\title{
Desenvolvimento de um obturador de feixe óptico utilizando um disco rígido de computador
}

\author{
The development of an optical shutter from a computer hard disk
}

\author{
N. B. Tomazio*1, A. L. S. Romero ${ }^{1}$, C. R. Mendonca ${ }^{1}$ \\ ${ }^{1}$ Universidade de São Paulo, Instituto de Física de São Carlos, São Carlos, SP, Brasil
}

Recebido em 19 de Junho, 2017. Revisado em 13 de Julho, 2017. Aceito em 15 de Julho, 2017.

\begin{abstract}
O objetivo deste trabalho é demonstrar a confecção de um obturador de feixe óptico a partir de um disco rígido de computador. O controle de corrente do dispositivo é feito por modulação de largura de pulso (Pulse width modulation - $P(W M)$, uma técnica que permite variar níveis de tensão/corrente por meio do ajuste da largura de pulso de um sinal digital. Este controle é feito de forma a minimizar a potência dissipada no dispositivo, aumentando assim sua vida útil. O núcleo do circuito de controle de corrente é o Arduino Uno, uma placa de microcontrolador de baixo custo e de fácil programação, amplamente utilizado como plataforma para prototipagem eletrônica. Por fim, a título de ilustração, apresentamos o uso do obturador de feixe óptico desenvolvido em um sistema de microfabricação via fotopolimerização por absorção de dois fótons, no qual ele tem papel fundamental. Palavras-chave: Obturador de feixe óptico, modulação por largura de pulso, Arduino UNO, fotopolimerização por absorção de dois fótons.
\end{abstract}

\begin{abstract}
This work demonstrates the development of an optical shutter from a computer hard disk. The electrical current control of the device is made by pulse width modulation, a technique that allows varying current/voltage levels by adjusting the pulse width of a digital signal. This control is made in order to minimize the power dissipated into the device, thus raising its lifetime. The main unit of the control circuit is Arduino Uno, a low cost and ease of programming microcontroller board, extensively used as a platform for electronic prototyping. As an example, we present the use of an optical shutter developed in a microfabrication setup via two-photon polymerization, in which it plays an important role.
\end{abstract}

Keywords: Optical shutter, pulse width modulation, Arduino Uno, two-photon polymerization.

\section{Introdução}

O obturador de feixe óptico é um acessório fundamental para montagens experimentais que têm o laser como componente central, e.g. fabricação a laser e microscopia, devido a sua função de bloquear o feixe de forma controlada. Além das opções disponíveis comercialmente, pode-se obter um obturador a partir de materiais comuns presentes num laboratório de pesquisa. O obturador apresentado neste trabalho foi construído a partir do braço de leitura/escrita de uma unidade de disco rígido de computador. Para que o feixe seja devidamente bloqueado, o braço de leitura/escrita de dados, convertido em elemento obturador, deve ser acionado de forma rápida. Para tanto, é necessária a aplicação de correntes elétricas altas que levam à dissipação térmica no dispositivo, podendo danificá-lo sob uso contínuo.

Com o intuito de evitar problemas associados ao superaquecimento do obturador, propomos o uso da técnica de modulação de largura de pulso $(P W M)$ para efetuar o controle da corrente elétrica aplicada ao braço obturador. Esta técnica consiste na geração de sinais analógicos

*Endereço de correspondência: crmendon@ifsc.usp.br através da modulação temporal de um sinal digital e vem sendo utilizada de forma eficiente para diversas aplicações, e.g. controle de iluminação de LEDs e do movimento de servomotores[1]. Para aplicar à técnica de $P W M$, foi utilizada a plataforma de prototipagem eletrônica Arduino[2]. Esta plataforma tem sido amplamente utilizada em instrumentação eletrônica por seu baixo custo e flexibilidade de aplicações. Além de apresentar boa capacidade de processamento e diversidade de recursos e funções, o Arduino também possui uma interface de programação bem intuitiva que o torna adequado para fins didáticos

\section{Detalhes da Construção}

Uma unidade de disco rígido de computador é composta basicamente de uma bobina, um par de ímãs permanentes, um braço de escrita/leitura de dados e um disco magnético girante, no qual as informações são gravadas. No caso particular da utilização do disco rígido como obturador de feixe óptico, seu disco magnético girante é removido. Para o acionamento do braço de escrita/leitura de dados, utilizado aqui como elemento obturador, é 
necessária a aplicação de uma corrente elétrica à bobina. Quando é desenergizado, o braço obturador volta à posição original por meio da ação de uma mola adicionada ao dispositivo. A Fig. 1 mostra um típico disco rígido de computador (a) e um disco rígido adaptado para uso como obturador de feixe óptico (b).

O procedimento para efetuar um controle eficiente de corrente elétrica do obturador se dá em dois estágios. Primeiramente, aplicamos um nível de corrente necessário para o acionamento rápido do braço obturador. Passado o tempo de resposta do dispositivo, ajustamos o nível de corrente para um valor mínimo que o mantém acionado. A corrente elétrica necessária para o acionamento rápido do nosso obturador é $570 \mathrm{~mA}$. Contudo, para manter seu acionamento pouco menos da metade desta corrente é suficiente, cerca de $200 \mathrm{~mA}$. Com a corrente reduzida pela metade da requerida para o acionamento rápido, a potência dissipada na bobina é 4 vezes menor que a potência de acionamento, o que representa uma queda acentuada no calor gerado no dispositivo. O tempo de resposta do obturador resultado de sua inércia mecânica e posicionamento em relação ao feixe óptico, precisa ser considerado neste controle para que o dispositivo seja devidamente acionado. Para medi-lo, o obturador é posicionado entre um laser de HeNe e um fotodiodo; e o atraso entre o sinal elétrico de acionamento e a saída do fotodiodo é medido com auxílio de um osciloscópio digital.

Este controle de corrente foi feito por meio da técnica de $P W M$. Esta técnica faz uso de um controle digital, o qual produz um sinal que oscila entre dois níveis de tensão, 0 e 5 V. A fração de tempo em que o sinal passa no nível de $5 \mathrm{~V}$ é chamada de largura de pulso

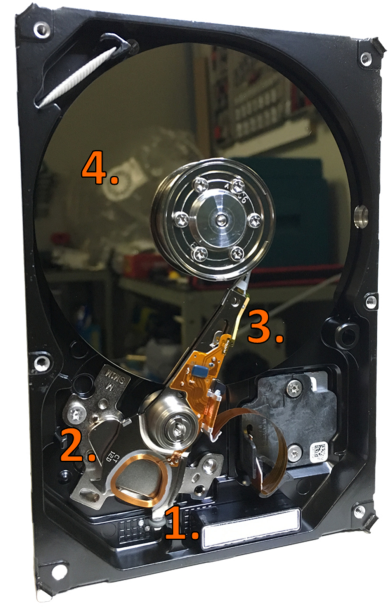

(a)

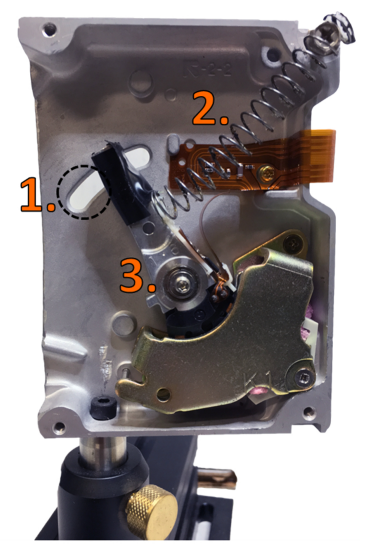

(b)
Figura 1: (a) Fotografia de um disco rígido de computador. Os números se referem a diferentes componentes do dispositivo: 1. Bobina, 2. Ímã permanente, 3. Braço de escrita/leitura de dados, 4. Disco magnético girante. (b) Fotografia de um disco rígido convertido em obturador de feixe óptico. 1. Fenda para exposição do feixe, 2. Mola, 3. Braço de escrita/leitura de dados. (duty cicle). Sua modulação permite o ajuste do nível de tensão/corrente aplicada ao dispositivo, resultado da média temporal do sinal digital. A título de ilustração, na Fig. 2 são mostrados sinais digitais com diferentes valores de duty cicle. A média temporal destes sinais resulta em níveis de tensão de $0.5,2.5$ e $3.75 \mathrm{~V}$, respectivamente.

O circuito elétrico utilizado neste projeto está esquematizado na Fig. 3. Sua alimentação é feita por uma fonte externa (eliminador de pilhas de 1 ampere), sendo seu componente principal o ATtiny85, um microcontrolador de baixo custo e pequenas dimensões. Ele está acoplado a uma placa Arduino Uno que intermedia sua comunicação com o ambiente de desenvolvimento integrado (IDE do inglês Integrated Development Environment) do Arduino, onde se dá sua programação. Por meio do pino 7, o microcontrolador faz a leitura do status do módulo de aquisição (trigger) que dá o comando para a abertura/fechamento do obturador. O sinal de trigger pode, por exemplo, ser gerado por um programa escrito na plataforma LabVIEW e enviado ao circuito por meio dos módulos de controle da National Instruments. Os pulsos de $P W M$ são sintetizados e enviados através do pino 5 ao transistor Q1, componente responsável pelo controle da corrente aplicada ao obturador. O potenciômetro P1 foi inserido no circuito para efetuar o ajuste da corrente mínima para o acionamento do obturador. Os demais componentes são adicionados para conter o ruído do sinal elétrico e garantir a segurança do obturador. Depois de programado, o microcontrolador funciona independentemente, permitindo assim que a montagem do circuito seja feita no próprio obturador, em uma placa protótipo parafusada em sua estrutura.

O programa gravado no microcontrolador foi escrito em linguagem $\mathrm{C}++$. Ele foi desenvolvido na IDE do Arduino,
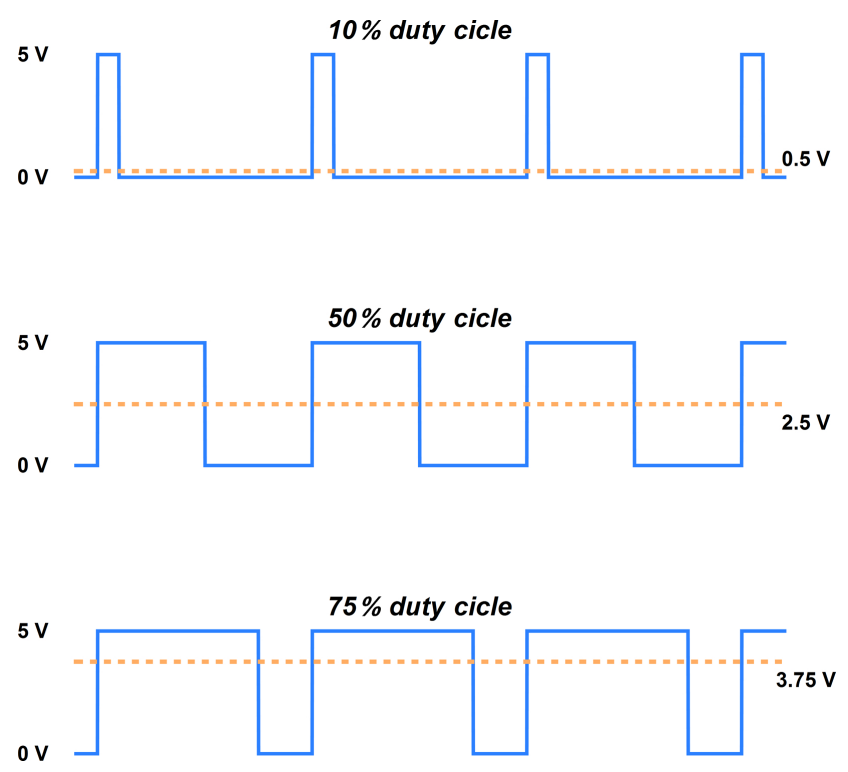

Figura 2: Sinais digitais com diferentes duty cicles. A linha tracejada em laranja representa a voltagem resultante da média temporal de cada sinal digital. 


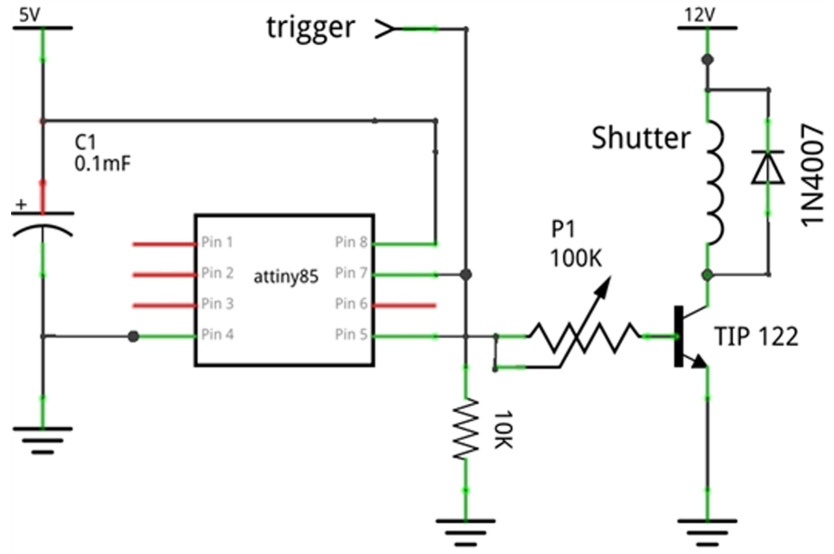

Figura 3: Diagrama esquemático do circuito (o termo Shutter se refere ao obturador).

que conta com um compilador integrado e conectividade com os kits de desenvolvimento dos microcontroladores. Inicialmente é necessário configurá-lo, definindo variáveis e indicando os pinos para leitura/escrita de dados:

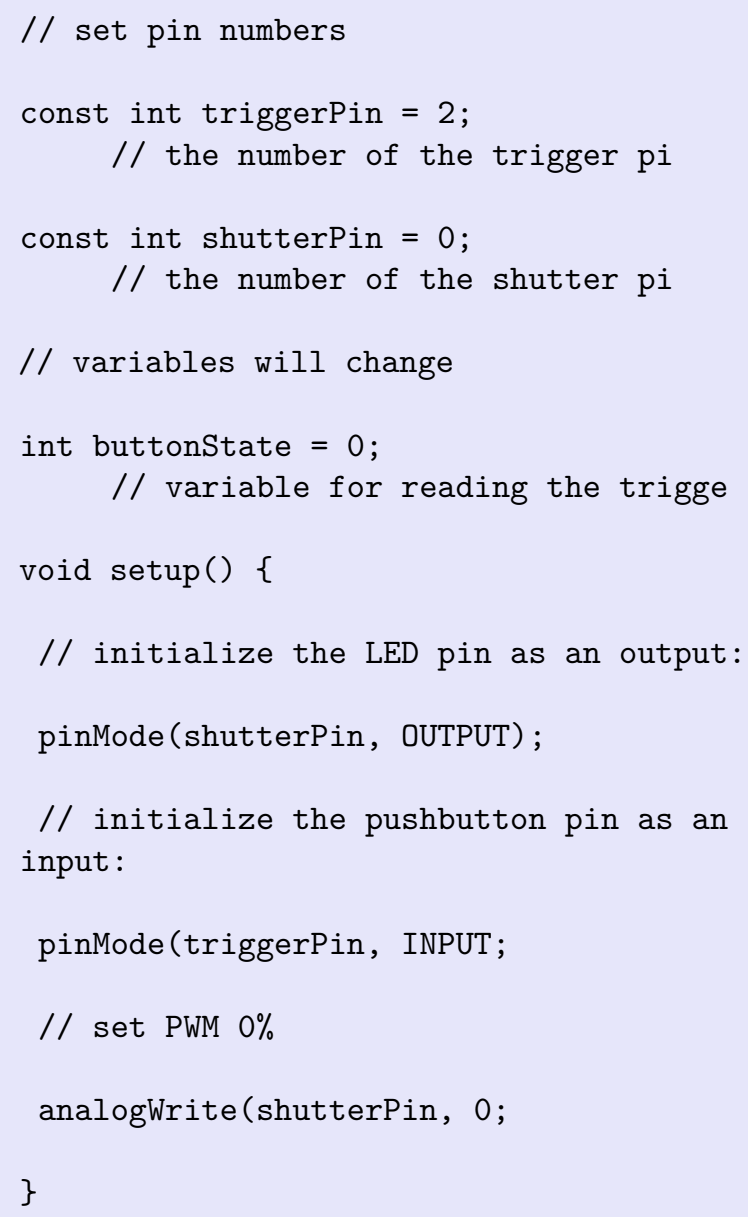

Depois de configurado, o programa aguarda a resposta da variável triggerpin, a qual recebe o sinal digital vindo do módulo de aquisição. Este sinal oscila entre dois níveis de tensão, o High (5 V) e o Low (0 V), conforme é mostrado na Fig. 4. Quando a resposta da variável triggerpin

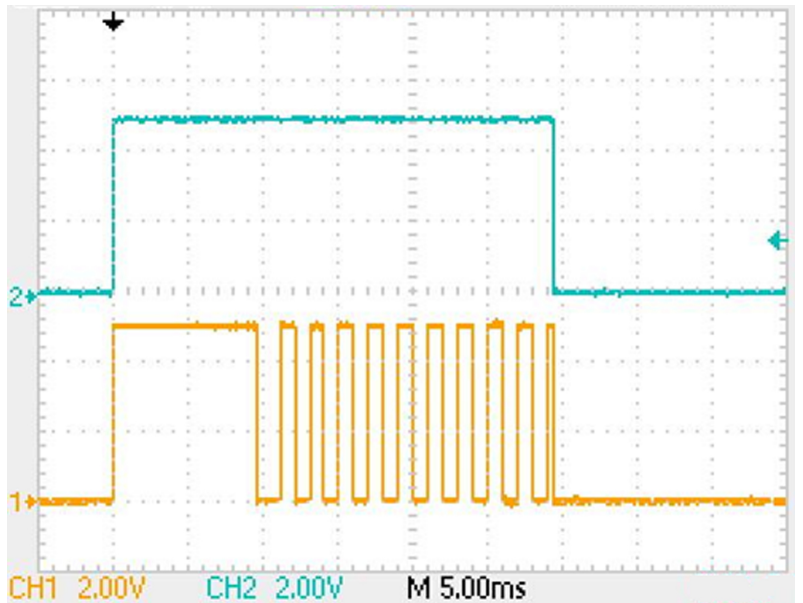

Figura 4: Sinais elétricos do sistema medidos por meio de um osciloscópio digital. Canal 1: Pulsos de PWM gerados pelo microcontrolador. Canal 2: Sinal digital de controle da rotina de abertura/fechamento do obturador (trigger).

for High, o programa ajusta o nível de $P W M$ para $100 \%$, aguarda $20 \mathrm{~ms}$ (tempo de resposta do obturador) e desce o nível de $P W M$ para $50 \%$. Em seguida, o programa fica em estado de espera até que a variável triggerpin seja alterada para Low. Nesta condição, o programa ajusta o nível de $P W M$ para $0 \%$ e repete a sequência de instruções:

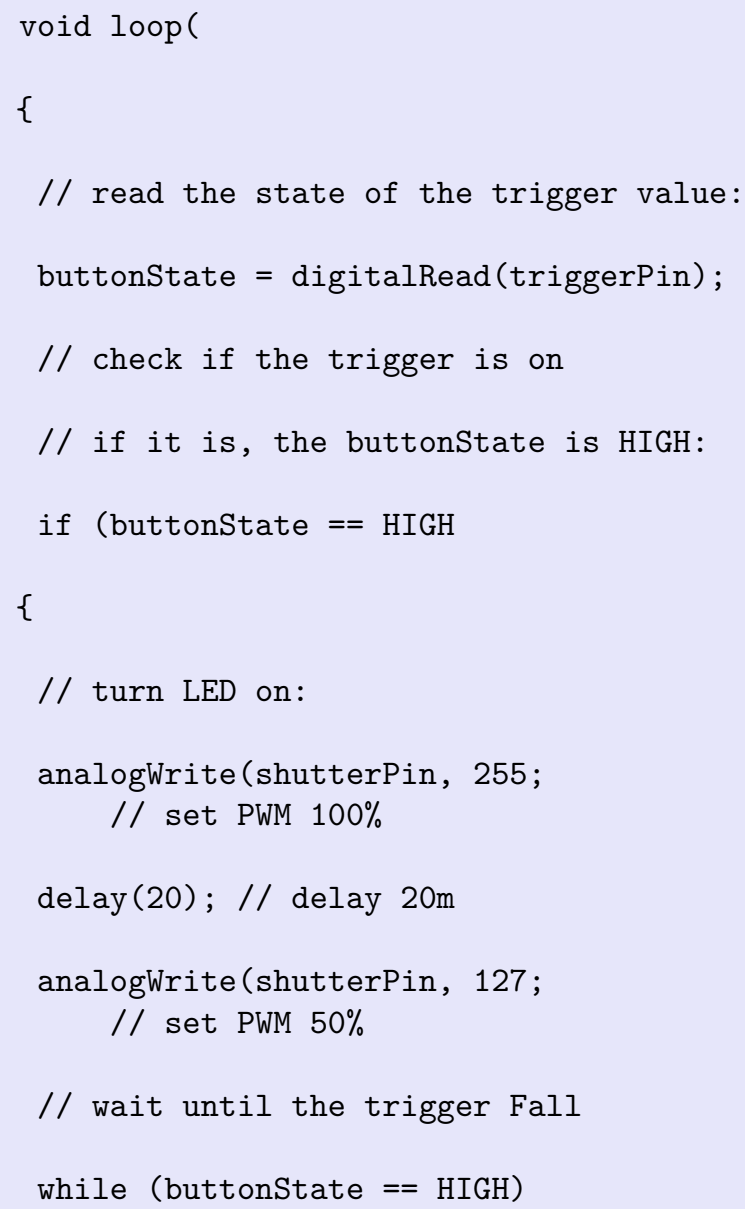




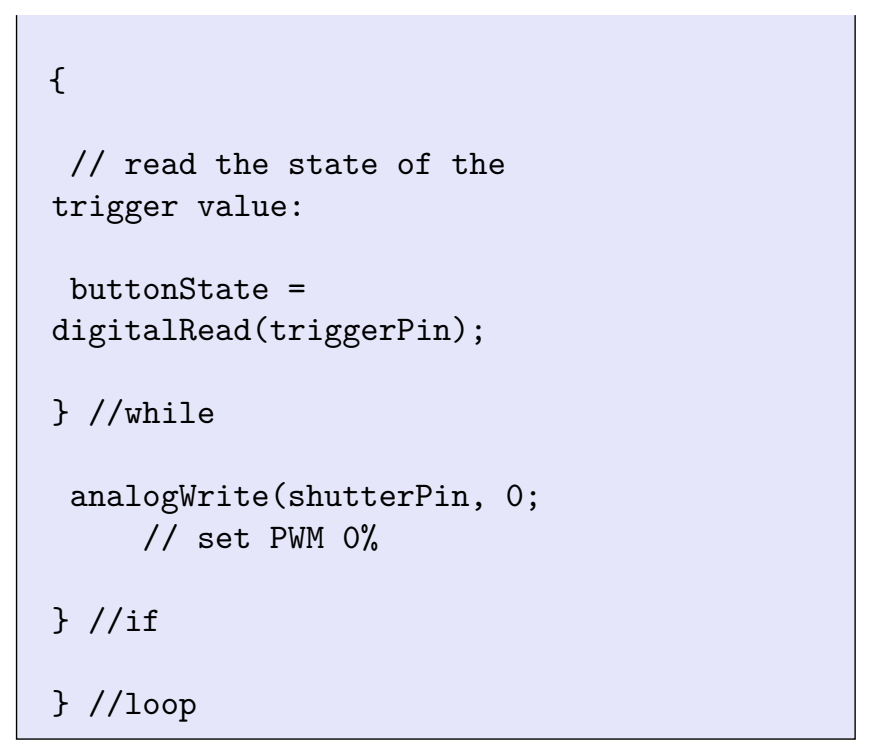

\section{Aplicação}

Uma das aplicações do obturador é controlar a exposição do feixe laser durante o processo de fotopolimerização por absorção de dois fótons (FA2F).[3] Esta técnica de microfabricação tem se destacado no meio científico devido a sua capacidade de produzir microestruturas tridimensionais de alta complexidade e variedade de formas, permitindo até mesmo a confecção de estruturas contendo partes móveis.[4,5] A FA2F consiste em focalizar um feixe pulsado de femtossegundos no volume de uma resina polimérica contendo um fotoiniciador, composto orgânico responsável por desencadear a reação de polimerização quando excitado via absorção de dois fótons.[6] Diferente do que ocorre no processo de absorção linear, a taxa na qual a absorção de dois fótons ocorre é proporcional à intensidade da radiação ao quadrado. Esta dependência não linear com a intensidade da radiação é a característica responsável pelo confinamento espacial da excitação, que confere à técnica resolução abaixo do limite de difração.[7] Ademais, diferentes polímeros podem ser utilizados para a fabricação das microestruturas, tornando a técnica viável para uma vasta gama de aplicações.

O aparato experimental para a microfabricação via FA2P está ilustrado na Fig. 5. O feixe proveniente de um oscilador laser de Ti:safira é direcionado a um par de espelhos galvanométricos, responsáveis pelo deslocamento do feixe no plano x-y da amostra. Ele é focalizado no volume da resina polimérica com auxílio de uma objetiva de microscópio. A resina é posicionada sobre um estágio de translação em z, o qual, em conjunto com os espelhos galvanométricos, permite deslocar o foco do feixe tridimensionalmente. O ajuste de intensidade do feixe laser é feito através de um polarizador e uma lâmina de meiaonda. O obturador, desenvolvido a partir de um disco rígido de computador conforme descrito anteriormente, é adicionado à montagem para controlar a exposição do feixe na resina e assim, evitar traçados de polimerização

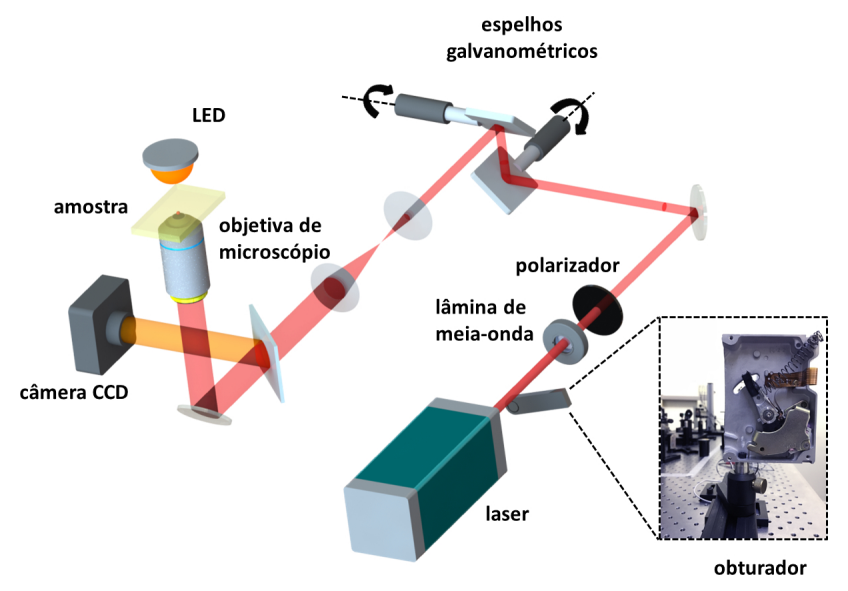

Figura 5: Aparato experimental utilizado para a microfabricação via FA2F, destacando os principais componentes do sistema.

indesejados que configuram defeitos de fabricação. O controle da abertura/fechamento do obturador, assim como do movimento dos espelhos galvanométricos e do estágio de translação em z, é realizado por meio de um único programa de computador. Todo o processo é monitorado em tempo real com auxílio de um sistema de iluminação formado por um LED e uma câmera CCD. A título de exemplo, são mostradas na Fig. 6 imagens de microscopia eletrônica de varredura de estruturas típicas produzidas por meio deste aparato de microfabricação.

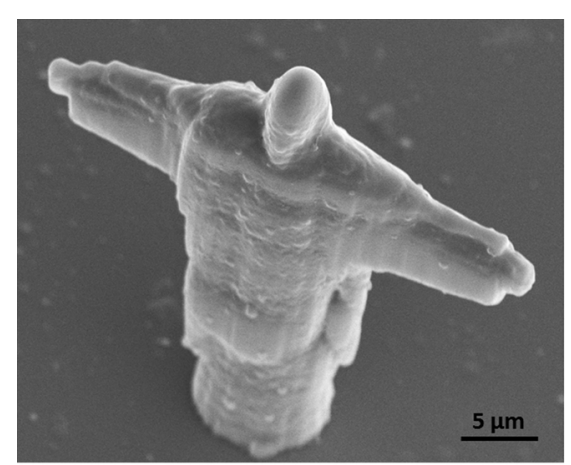

(a)

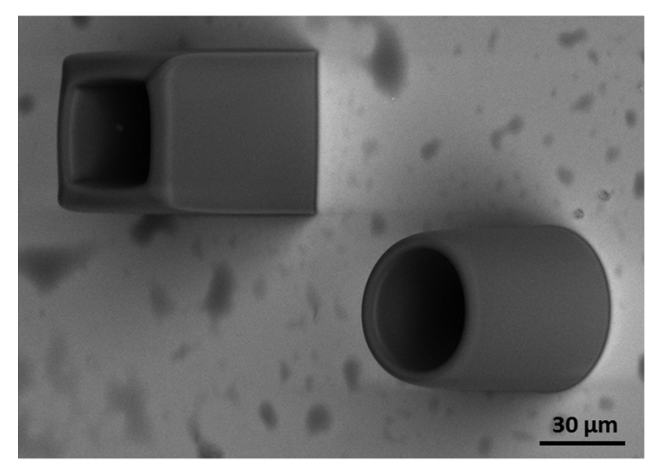

(b)

Figura 6: Microestruturas fabricadas pelo nosso sistema de microfabricação via FA2F. (a) Cristo Redentor (b) Cilindro e cubo ocos. 


\section{Conclusão}

O obturador de feixe óptico apresentado neste trabalho foi confeccionado a partir de um disco rígido de computador, que pode facilmente ser obtido em um laboratório de ensino/pesquisa. Para efetuar um controle eficiente de corrente elétrica aplicada ao obturador, utilizamos a plataforma de prototipagem eletrônica Arduino Uno, que além de barata e flexível, dispõe de uma interface de programação simples, adequada para o uso de pessoas com pouca experiência em eletrônica. Este controle de corrente, feito por meio da técnica de modulação de largura de pulso, permite uma queda significativa de dissipação térmica no dispositivo, contribuindo assim para prolongar sua vida útil. Todos os detalhes da construção do circuito e programação foram explicados em detalhe para que outros estudantes possam reproduzi-lo. Para demonstrar o uso do obturador foi apresentada a fotopolimerização por absorção de dois fótons, uma técnica de microfabricação poderosa que permite o processamento de estruturas tridimensionais de alta resolução e com flexibilidade de materiais e geometrias.

\section{Agradecimentos}

Gostaríamos de agradecer ao Conselho Nacional de Desenvolvimento Científico e Tecnológico (CNPq), à Coordenação de Aperfeiçoamento de Pessoal de Nível Superior (CAPES) e à Fundação de Amparo à Pesquisa do Estado de São Paulo (FAPESP) pelo suporte financeiro, ao Instituto de Física de São Carlos e ao Grupo de Fotônica pela infraestrutura necessária para o desenvolvimento deste projeto e ao aluno de doutorado Gustavo Foresto Brito de Almeida e ao Dr. Adriano José Galvani Otuka pela fabricação da microestrutura em formato de Cristo Redentor.

\section{Referências}

[1] J. Arrillaga, Y.H. Liu and N.R. Watson, Flexible Power Transmission: The HVDC Options (John Wiley \& Sons, Hoboken, 2007).

[2] Arduino homepage, https://www.arduino.cc, acesso em 26/04/2017.

[3] S. Maruo, O. Nakamura and S. Kawata, Optics Letters 22, 132 (1997).

[4] S. Maruo, K. Ikuta and H. Korogi, Journal of Microelectromechanical Systems 12, 533 (2003).

[5] A. Ostendorf and B.N. Chichkov, Photonics Spectra 40, $72(2006)$.

[6] C.N. Lafratta, J.T. Fourkas, T. Baldacchini and R.A. Farrer, Angewandte Chemie-International Edition 46, 6238 (2007).

[7] S. Maruo and J.T. Fourkas, Laser \& Photonics Reviews 2, 100 (2008). 